\title{
Modeling and Control of a New Robotic Deburring System
}

\author{
Jae H. Chung \\ US Army RDECOM-ARDEC \\ Building $95 \mathrm{~N}$ \\ Picatinny Arsenal, NJ 07806
}

USA

\section{Introduction}

A machining manipulator is subject to mechanical interaction with the object being processed. The robot performs the task in constrained work space. In constrained tasks, one is concerned with not only the position of the robot end-point, but also the contact forces, which are desired to be accommodated rather than resisted. Therefore, interaction force needs to be considered in designing and controlling deburring tools.

Many researchers have proposed automated systems for grinding dies, deburring casting, removing weld beans, etc [Bopp, 1983; Gustaffson, 1983]. Usually, a deburring tool is mounted on a NC machining center or a robot manipulator. Several control laws have been developed for simultaneous control of both motion and force [Whitney, 1987; Hogan, 1984] of robotic manipulators. Despite the diversity of approaches, it is possible to classify most of the control methods into two major approaches: impedance control [Wang \& Cheah, 1996; Carelli \& Kelly, 1991] and hybrid position/force control [Raibert \& Craig, 1981; Yoshikawa et al., 1988]. However, these methods require an accurate model of force interaction between the manipulator and the environment and are difficult to implement on typical industrial manipulators that are designed for position control.

An active feedback control scheme was developed in order to supply compliance for robotic deburring as a means to accommodate the interaction force due to contact motion. Kuntze [Kuntze, 1984] suggested an active control scheme, in which the actuators are commanded to increase torques in the opposite direction of the deflections. Paul [Paul et al., 1982] applied an active isolator to a chipping robot, where the isolator attached to the arm tip reduces the vibration seen by the robot. Sharon and Hardt [Sharon and Hardt, 1984] developed a multi-axis local actuator, which compensates for positioning errors at the end point, in a limited range.

Asada [Asada \& Sawada, 1984] developed passive tool support mechanisms, which couple the arm tip to the workpiece surface and bear large vibratory loads. These mechanisms allow the robot to compensate for the excessive deflection when the robot contacts the workpiece. These methods reduce dynamic deflection in a certain frequency range. However, it is difficult for these control schemes, which are employed for a robot with a 
passive tool, to perform well over a wide frequency band because they must drive the entire, massive robot arm. In addition, unknown compliance from a passive tool makes it difficult to control the deburring robot.

In this paper, a robotic deburring method is developed based on an integrated pneumatic actuation system (IPAS), which considers the interaction among the tool, the manipulator, and the workpiece and couples the tool dynamics and a control design that explicitly considers deburring process information. A new active tool is developed based on two pneumatic actuators, which utilizes double cutting action - initial cut followed by fine cut. Then, a coordination based control method is developed for the robotic deburring system based on the active pneumatic deburring tool. The developed control method employs a hierarchical control structure based on a coordination scheme. Robust feedback linearization is utilized to minimize the restrained elements of the precision deburring such as static and Coulomb friction and nonlinear compliance of the pneumatic cylinder stemming from the compressibility of air.

\section{Modeling of the Deburring Robot}

In this section, a dynamic model of a robotic arm with the new deburring tool or IPAS is developed as a robotic deburring system as shown in Fig. 2. Fig. 1 shows the integrated cylinder, which is comprised of three chambers and actuated by a single valve connected to Chamber 3. Note that the IPAS is a single input system with two pistons. The pistons are not directly connected to the inner pistons, $M_{t 3}$ and $M_{t 4}$, which create a unique configuration of three chambers connected in series. This configuration allows the chambers adjacent to the active chamber to act as vibration isolators. This feature enables the IPAS to damp out the chatter caused by external loads and air compressibility. Therefore, a double cutting action and chattering reduction can be achieved simultaneously.
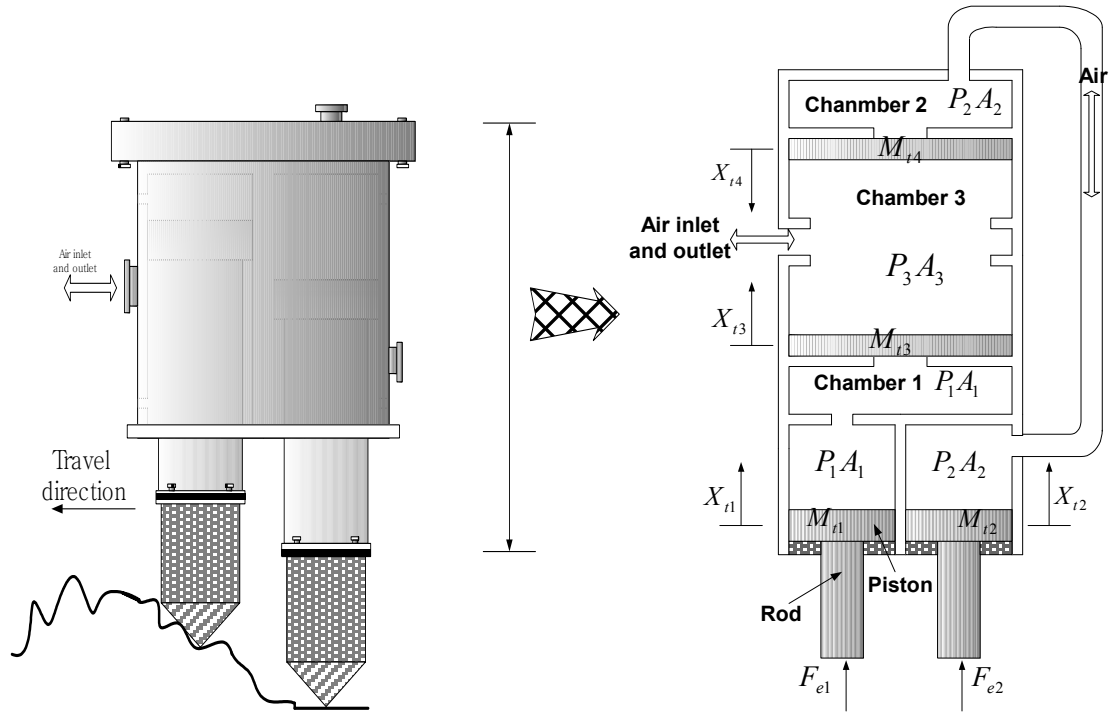

Fig. 1. Integrated double cylinder system 
The dynamics of the chambers can be written as [Sorli et al., 1999]

$$
G_{3}=\rho_{3} \frac{d V_{3}}{d t}+V_{3} \frac{d \rho_{3}}{d t}
$$

where $G_{3}$ is the entering air flow, $\rho_{3}$ the air density and $V_{3}$ the volume of Chamber 3. It is assumed that the condition of the air is ideal as following:

$$
\rho_{3}=\rho_{3 j}\left(\frac{P_{3}}{P_{3 j}}\right)^{1 / n}=\frac{P_{3 j}}{R T_{3 j}}\left(\frac{P_{3}}{P_{3 j}}\right)^{1 / n}
$$

where the subscript $j$ indicates the initial conditions and $n$ is the air transformation ratio. Now, $V_{3}$ is derived as

$$
V_{3}=A_{3}\left(L-X_{t 4}-X_{t 3}\right)
$$

where $A_{3}$ denotes the area of Piston 3 , and $X_{t i}(i=4,3)$ is the position of Piston $i . L$ denotes the length of Chamber 3 as shown in Fig. 3. By combining Eqs. (2) and (3) and their time derivatives in Eq. (1), the following expression is be obtained:

$$
\begin{aligned}
& G_{3}=A_{3}\left(L-X_{t 3}\right) \frac{1}{n R T_{3 j}}\left(\frac{P_{3}}{P_{3 j}}\right)^{1 / n-1} \frac{d P_{3}}{d t}+\frac{P_{3 j}}{R T_{3 j}}\left(\frac{P_{3}}{P_{3 j}}\right)^{1 / n} A_{3} \frac{d X_{t 3}}{d t} \\
& +A_{3}\left(L-X_{t 4}\right) \frac{1}{n R T_{3 j}}\left(\frac{P_{3}}{P_{3 j}}\right)^{1 / n-1} \frac{d P_{3}}{d t}+\frac{P_{3 j}}{R T_{3 j}}\left(\frac{P_{3}}{P_{3 j}}\right)^{1 / n} A_{3} \frac{d X_{t 4}}{d t}
\end{aligned}
$$

Then, the pressure gradient is be written as

$$
\begin{aligned}
& \frac{d P_{3}}{d t}=\frac{n R T_{3 j}}{A_{3}\left(L-X_{t 3}\right)\left(P_{3} / P_{3 j}\right)^{1 / n-1}} G_{3}-\frac{n P_{3}}{\left(L-X_{t 3}\right)} \frac{d X_{t 3}}{d t} \\
& +\frac{n R T_{3 j}}{A_{3}\left(L-X_{t 4}\right)\left(P_{3} / P_{3 j}\right)^{1 / n-1}} G_{3}-\frac{n P_{3}}{\left(L-X_{t 4}\right)} \frac{d X_{t 4}}{d t}
\end{aligned} .
$$

The dynamic equations are written as

$$
\left[\begin{array}{cc}
M_{t 3} & 0 \\
0 & M_{t 4}
\end{array}\right]\left[\begin{array}{l}
\ddot{X}_{t 3} \\
\ddot{X}_{t 4}
\end{array}\right]+C\left[\begin{array}{l}
\left(\dot{X}_{t 1}-\dot{X}_{t 3}\right) \\
\left(\dot{X}_{t 1}-\dot{X}_{t 4}\right)
\end{array}\right]+K\left[\begin{array}{l}
\left(X_{t 1}-X_{t 3}\right) \\
\left(X_{t 1}-X_{t 4}\right)
\end{array}\right]=\left[\begin{array}{c}
P_{3} A_{3}-F_{f 3} \\
P_{3} A_{3}-F_{f 4}
\end{array}\right]
$$

where $K$ and $C$ are the stiffness and damping coefficients of the system, respectively, $\dot{X}_{t i}$ and $\ddot{X}_{t i}$ represent the velocity and the acceleration of each piston $(i=1,2,3,4) . F_{f i}$ denotes the viscous friction force of the piston $\operatorname{rod}(i=1,2,3,4), F_{e i}$ is the external force $(i=1,2), P_{i}$ and $A_{i} \quad(i=1,2,3)$ denote the air pressure and the area of the piston, respectively, and $M_{t 1}$ and $M_{t 2}$ are the masses of each position rod.

\subsection{Robotic Deburring System}

Fig. 2 illustrates a multi-link rigid robot with the pneumatic deburring tool described earlier. Using the well-known Lagrangian equations, the following equations of motion of the deburring robot can be obtained: 


$$
\bar{m}(q) \ddot{q}+\bar{c}(q, \dot{q}) \dot{q}+\bar{g}(q)=\tau
$$

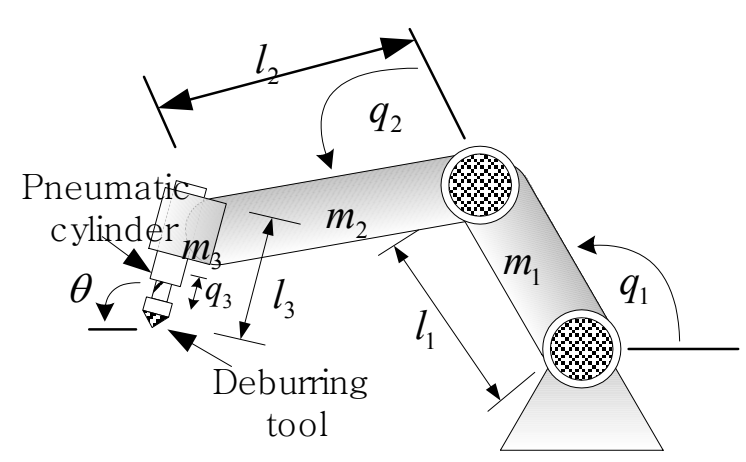

Fig. 2. Deburring robot with pneumatic tool

where $q, \dot{q}, \ddot{q}$ are the joint angle, the joint angular velocity, and the joint angular acceleration, respectively, $\bar{m}(q)$ is the $3 \times 3$ symmetric positive-definite inertia matrix, $\bar{c}(q, \dot{q}) \dot{q}$ is the $3 \times 1$ vector of Coriolis and centrifugal torques, $\bar{g}(q)$ is the $3 \times 1$ gravitational torques, and $\tau$ is the $3 \times 1$ vector of the joint torques.

The mass of the links and pneumatic cylinder are considered as if they were rigidly attached. The relationship between the joint and the tip velocities can be written as

$$
\dot{x}=J(q) \dot{q}
$$

where $J(q)$ is the geometric Jacobian of the manipulator. By differentiating Eq. (8), the Cartesian acceleration term can be found as

$$
\ddot{x}=J(q) \ddot{q}+\dot{J} \dot{q}
$$

Then, the equations of motion of the robot are obtained as following:

$$
m(x) \ddot{x}+c(x, \dot{x})+g(x)=f
$$

where $f=\left(J^{T}\right)^{-1} \tau$ is input expressed in task space and $m(x)$ is the inertia matrix, $c(x, \dot{x})$ is Coriolis and centrifugal forces, and $g(x)$ is gravitational forces.

Let the dynamic equation of the robot manipulator in the constraint coordinates be represented as

$$
m(x) \ddot{x}+c(x, \dot{x}) \dot{x}+g(x)=f+f_{r f}
$$

where $f$ denotes the input force and $f_{r f}$ is the resultant force of the normal force $f_{n}$ and the tangential force $f_{t}$ exerted on the tool tip. The tangential force [18] can be represented as

$$
f_{t}=\frac{b d v_{t} e_{m}}{V_{t}}
$$


where $V_{t}$ is the spindle speed of deburring tool; $b$ is the tool width; $d$ is the depth of cut; $v_{t}$ is the feed rate (or the traveling speed of the end effector along the surface of the workpiece); $e_{m}$ is the material-stiffness of the workpiece. The normal force $f_{n}$ is assumed to be proportional to the tangential force $f_{t}$. Besides, the force angle of the deburring tool affects the tangential force. Although the value of the angle may vary substantially depending on the nature of the material flow at the tool-chip interface, as approximation 0.3 was used in these calculations [Raibert \& Craig, 1981]. Therefore, the normal force $f_{n}$ is considered to be smaller than the tangential force $f_{t}$ in Eq. (12), where the ratio is $f_{n} / f_{t} \approx 0.3$ [Deccusse \& Moog, 1985].

\section{Control Design}

The IPAS based deburring robot can be treated as a system that consists of two primary subsystems; the arm and the IPAS. The two subsystems differ substantially in their task assignments, dynamic characteristics and controller requirements. This physical interpretation provides an efficient approach to the control of the robotic deburring system. The control strategy for the deburring robot is illustrated in Fig. 3. The arm is commanded to follow the desired trajectory in task space, which is modified based on the position of the second piston due to varying length of the tool. In other words, the primary cutter at the front side cuts the burr first and the second cutter then attempts to eliminate the remaining burr. In case that the burr is not removed completely, the uncut depth is incorporated into the desired trajectory for compensation.

The developed control design is a decentralized control [Deccusse \& Moog, 1985; Isidori, 1985], which consists of two independent controllers interacting based on the coordination scheme aforementioned for the manipulator and the IPAS, respectively. Constraint equations are derived in terms of position variables and are differentiated twice to lead to a relationship in terms accelerations, which integrate the separate controllers for stability proof. Feedback linearization is employed to design a coordination based controller. In what follows, it is shown that use of a nonlinear dynamic feedback achieves exact linearization and input-output decoupling for the robotic deburring system. However, pneumatic actuators typically have a limited bandwidth restricting the high gains which can be applied. Combined with their limited damping and low stiffness properties, which arise from the compressibility of air, the accuracy and repeatability of the performance can be limited under variable payload and supply pressure. Therefore, robust feedback linearization is employed to reduce the undesirable effect of nonlinear compliance of the pneumatic cylinder. The coordination control method is developed first and then its efficiency will be compared with the hybrid control method through simulation study.

\subsection{Coordination Control}

Shown in Fig. 3 is the control design for the deburring robot with the active pneumatic tool. Note that $X_{t}$ denotes the position of the piston, respectively relative to their origins as described in Section 2.1. The desired trajectories of the robot wrist, denoted as $x_{r}^{d}, \dot{x}_{r}^{d}, \ddot{x}_{r}^{d}$, are modified to compensate the uncut depth based on the position of the second piston due to 
the varying length of the tool. Additionally, $X_{t}^{d}, \dot{X}_{t}^{d}$, and $\ddot{X}_{t}^{d}$ denote the desired trajectories for the IPAS. Feedback linearization [Isidori, 1985] is employed to design a coordination based controller. In what follows, it is shown that the use of a nonlinear dynamic feedback achieves exact linearization and input-output decoupling for the robotic deburring system.

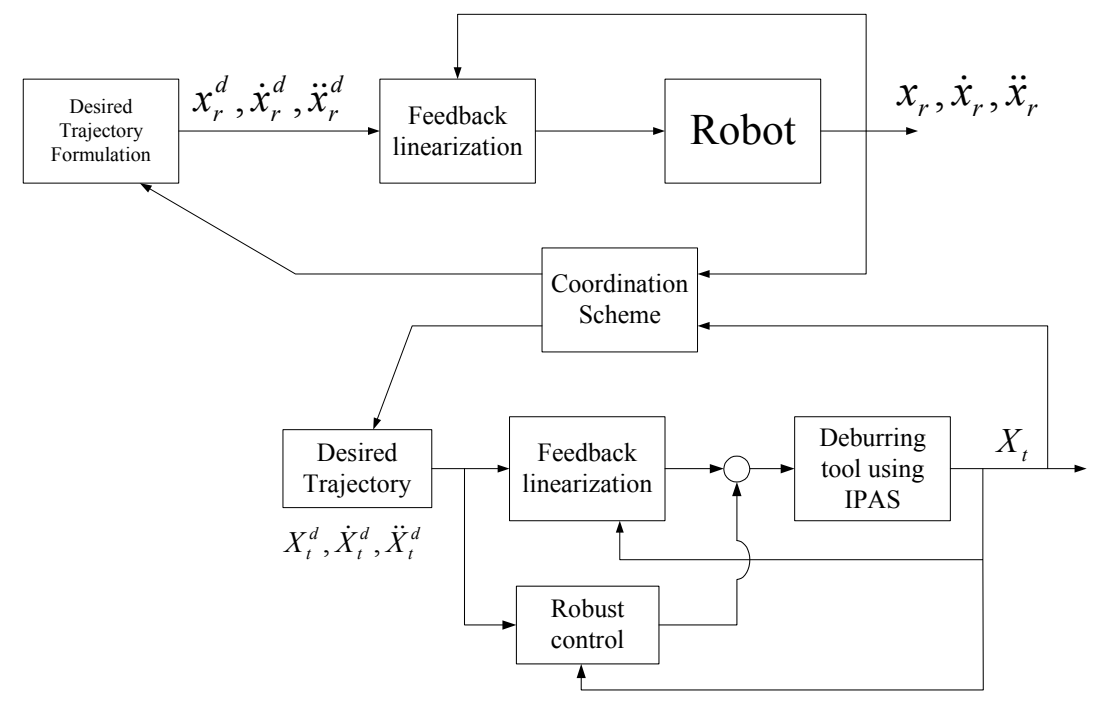

Fig. 3. Block diagram for coordinated control for robotic deburring

We assume that the robot has $\mathrm{n}$ links. The equations of motion of the arm are rewritten in a decentralized form as

$$
m_{r}\left(x_{r}\right) \ddot{x}_{r}+c_{r}\left(x_{r}, \dot{x}_{r}\right)=f_{r}-R_{r}\left(x_{r}\right) \ddot{X}_{t}
$$

where $x_{r}, \dot{x}_{r}$ and $\ddot{x}_{r}$ denote the displacement, velocity and acceleration matrix of the tip of the manipulator $n \times 1, m_{r}$ is the inertia mass matrix $n \times n, c_{r}$ is the matrix $n \times n$, which consists of Coriolis, centripetal, and gravity forces, $f_{r}$ is the input force matrix acting on the tip of the manipulator $n \times 1, R_{r}$ is the inertia matrix which reflects the dynamic effect of the deburring tool on the manipulator $n \times n$, and $\ddot{X}_{t}$ is the acceleration of IPAS $n \times 1$. Likewise, the equations of motion for deburring tool are written as

$$
M_{t} \ddot{X}_{t}-C_{t}\left(\dot{X}_{t}, D\left(X_{t}\right), X_{t}, \operatorname{sgn}\left(\dot{X}_{t}\right), \mu_{c}, \mu_{u}\right)+F_{e}=F_{t}(A, P)-R_{t}\left(x_{r}\right) \ddot{x}_{r}
$$

where $\ddot{X}_{t}$ and $\dot{X}_{t}$ denote the acceleration and velocity matrix $n \times 1$ of the tool, $M_{t}$ is the mass matrix $n \times n$ of the piston, $C_{t}$ is a polynomial function of the nonlinear term $n \times 1, \mu_{c}$ is Coulomb term, $\mu_{u}$ is viscous coefficient [11], $D\left(X_{t}\right)$ is a polynomial function of the nonlinear spring caused by air compression in Eq. (14), $F_{t}$ is the forces matrix $n \times 1$ acting 
on the piston, $R_{t}$ is the inertia matrix $n \times n$ which represents the end effect of the manipulator on the tool, $F_{e}$ is the external force matrix $n \times 1$ of the IPAS.

Let $p \in R^{m}$ denote the position vector of the tip of the robot in the fixed workspace coordinate system. The robotic deburring system is assumed to have the constraint surface defined in algebraic terms by

$$
\phi(p)=\left[\begin{array}{c}
\phi_{1}(p) \\
\vdots \\
\phi_{n+1}(p)
\end{array}\right]=0
$$

where $p$ is comprised of $x_{r}$ and $X_{t}$. Now, the constraint Eq. (15) is differentiated once as following:

$$
\dot{\phi}(p)=J_{c}(q) \dot{q}=0
$$

where $J_{c}$ denotes the geometric Jacobian matrix $n \times n$. The initial Lagrange coordinate $q_{0}$ satisfies the holonomic constraint $\phi\left(p_{0}\right)=0$, where $p_{0}$ is the initial position of the robot. Then, Eq. (16) is differentiated once to produce $\ddot{\phi}=0$, into which the subsystems, Eqs. (13) and (14) are incorporated. Then, feedback linearization can be applied to cancel the coupling terms and to design linear controllers as the outer feedback loop. Since the manipulator velocity is always in the null space of $\dot{\phi}(p)$, it is possible to define a vector of generalized velocities $\eta(t)$, which is the $n \times 1$ dimensional matrix as following:

$$
\dot{x}_{r}=\zeta\left(x_{r}\right) \eta(t)=\left[\begin{array}{c}
\dot{x}_{r 1} \\
\vdots \\
\dot{x}_{r n}
\end{array}\right]=\left[\begin{array}{ccc}
\zeta_{1}\left(x_{r 1}\right) & \cdots & 0 \\
\vdots & \ddots & \vdots \\
0 & \cdots & \zeta_{n}\left(x_{r n}\right)
\end{array}\right]\left[\begin{array}{c}
\eta_{1}\left(t_{1}\right) \\
\vdots \\
\eta_{n}\left(t_{n}\right)
\end{array}\right]
$$

where the columns of $\zeta\left(x_{r}\right)$ are in the $n \times n$ dimensional null space of $\phi(p)$. Differentiating Eq. (15), substituting the resulting expression for $\ddot{x}_{r}$ into Eq. (13), and premultiplying Eq. (13) by $\zeta^{T}$, we obtain

$$
\zeta^{T}\left(m_{r} \zeta \dot{\eta}+m_{r} \dot{\zeta} \eta+c_{r}\right)=\zeta^{T} f_{r}-\zeta^{T} R_{r} \ddot{X}_{t}
$$

Note that $\zeta^{T} \phi^{T}=0$. Similarly substituting $\ddot{x}_{r}$ into Eq. (14), we have

$$
M_{t} \ddot{X}_{t}-C_{t}+F_{e}=F_{t}-R_{t} \dot{\zeta} \eta-R_{t} \zeta \dot{\eta}
$$

Using the state vector $\chi=\left[\begin{array}{llll}x_{r}^{T} & X_{t}^{T} & \eta^{T} & \dot{X}_{t}^{T}\end{array}\right]^{T}$ and the block partition of the state vector 


$$
\chi=\left[\begin{array}{c}
\chi_{1} \\
\chi_{2} \\
\chi_{3}
\end{array}\right], \text { with } \chi_{1}=x_{r}=\left[\begin{array}{c}
x_{r 1} \\
\vdots \\
x_{r n}
\end{array}\right], \chi_{2}=X_{t}=\left[\begin{array}{c}
x_{t 1} \\
\vdots \\
x_{t n}
\end{array}\right], \chi_{3}=\left[\begin{array}{c}
\eta \\
\dot{X}_{t}
\end{array}\right]=\left[\begin{array}{c}
\eta_{1} \\
\vdots \\
\eta_{n} \\
\dot{X}_{t 1} \\
\vdots \\
\dot{X}_{t n}
\end{array}\right]
$$

the following expression is obtained:

$$
\dot{\chi}=\left[\begin{array}{c}
\dot{\chi}_{1} \\
\dot{\chi}_{2} \\
\dot{\chi}_{3}
\end{array}\right]=\left[\begin{array}{c}
\zeta \eta \\
\dot{X}_{t} \\
M^{-1} C
\end{array}\right]+\left[\begin{array}{c}
0 \\
0 \\
M^{-1} E
\end{array}\right] \lambda
$$

where

$$
M=\left[\begin{array}{cc}
\zeta^{T} m_{r} \zeta & \zeta^{T} R_{r} \\
R_{t} \zeta & M_{t}
\end{array}\right], E=\left[\begin{array}{cc}
\zeta^{T} & 0 \\
0 & I
\end{array}\right], C=\left[\begin{array}{cc}
-\zeta^{T} m_{r} \dot{\zeta} \eta & -\zeta^{T} c_{r} \\
C_{t}+F_{e} & -R_{t} \dot{\zeta} \eta
\end{array}\right]
$$

The system is input-output linearizable by using the following nonlinear feedback:

$$
\left[\begin{array}{c}
\lambda_{1} \\
\vdots \\
\lambda_{n}
\end{array}\right]=\left[\begin{array}{ccc}
E_{1}^{-1} & \cdots & 0 \\
\vdots & \ddots & \vdots \\
0 & \cdots & E_{1}^{-1}
\end{array}\right]\left\{\left[\begin{array}{ccc}
M_{1} & \cdots & 0 \\
\vdots & \ddots & \vdots \\
0 & \cdots & M_{n}
\end{array}\right]\left[\begin{array}{c}
u_{1} \\
\vdots \\
u_{n}
\end{array}\right]-\left[\begin{array}{c}
C_{1} \\
\vdots \\
C_{n}
\end{array}\right]\right\}
$$

which results in simpler state equations as following:

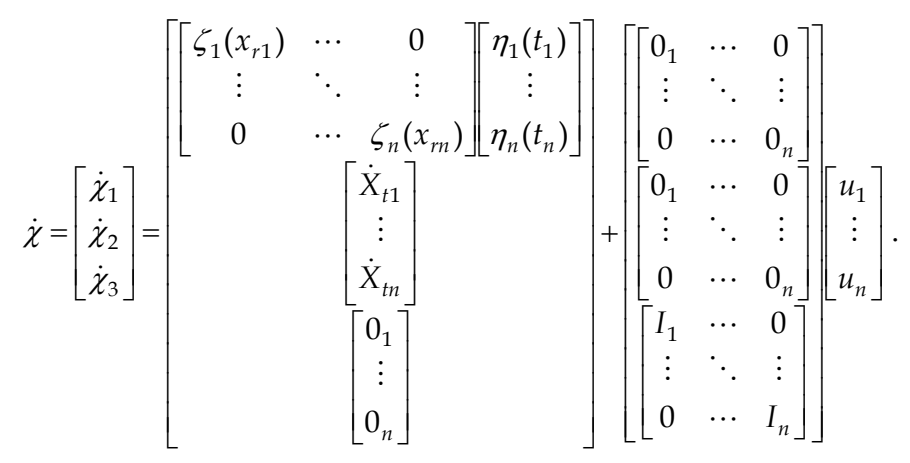

To derive the decoupling matrix, each component of the output equations is differentiated until the input appears explicitly in the derivative. In this case, the output equation is differentiated twice as following: 


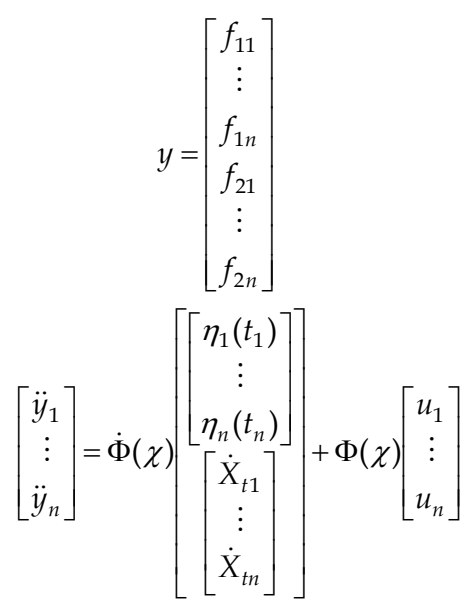

where $\Phi(\chi)$ is the decoupling matrix of the system given by

$$
\Phi(\chi)=\left[\begin{array}{cc}
\Phi_{r}(\chi) & 0 \\
0 & \Phi_{t}(\chi)
\end{array}\right]
$$

where

$$
\begin{gathered}
\Phi_{t}(\chi)=\left[\begin{array}{ccc}
\Phi_{t 1} & \cdots & 0 \\
\vdots & \ddots & \vdots \\
0 & \ldots & \Phi_{t n}
\end{array}\right]=\left[\begin{array}{ccc}
\frac{\partial f_{11}\left(X_{t 1}\right)}{\partial X_{t 1}} & \cdots & 0 \\
\vdots & \ddots & \vdots \\
0 & \cdots & \frac{\partial f_{1 n}\left(X_{t n}\right)}{\partial X_{t n}}
\end{array}\right], f_{1 n}=X_{t n}+f_{2 n} \\
\Phi_{r}(\chi)=\left[\begin{array}{ccc}
\Phi_{r 1} & \cdots & 0 \\
\vdots & \ddots & \vdots \\
0 & \ldots & \Phi_{r n}
\end{array}\right]=\left[\begin{array}{ccc}
f_{21} & \cdots & 0 \\
\vdots & \ddots & \vdots \\
0 & \cdots & f_{2 n}
\end{array}\right] .
\end{gathered}
$$

Applying the following nonlinear state feedback

$$
\left[\begin{array}{c}
u_{1} \\
\vdots \\
u_{n}
\end{array}\right]=\left[\begin{array}{cc}
\Phi_{r}(\chi) & 0 \\
0 & \Phi_{t}(\chi)
\end{array}\right]^{-1}\left(\left[\begin{array}{c}
v_{1} \\
v_{2}
\end{array}\right]-\left[\begin{array}{cc}
\dot{\Phi}_{r}(\chi) & 0 \\
0 & \dot{\Phi}_{t}(\chi)
\end{array}\right]\left[\begin{array}{c}
\eta \\
\dot{X}_{t}
\end{array}\right]\right),
$$

the input-output relationship is decoupled because each component of the auxiliary input, $v$, controls one and only one component of the output, $y$. It is noted that the existence of the nonlinear feedback require the inverse of the decoupling matrix $\Phi(\chi)$. To complete the controller design, it is necessary to stabilize each of the above subsystem with constant state feedback. Then, the stability of the system is guaranteed by selecting appropriate constant feedback gains for the linearized system.

Now, robust feedback linearization is employed to minimize the undesirable effect of external disturbances such as static and Coulomb friction and nonlinear compliance of the 
pneumatic cylinder stemming from the compressibility of air as appeared in Eq. (14). Let the tracking error be defined $e_{t}=X_{t}-X_{t}^{d}$. From Eq. (14) the following expression can be obtained: one obtains

$$
\ddot{X}_{t}=\frac{1}{M_{t}}\left(F_{t}(A, P)-R_{t}\left(x_{r}\right) \ddot{x}_{r}+C_{t}\left(\dot{X}_{t}, K_{S}\left(X_{t}\right), X_{t}, \operatorname{sgn}\left(\dot{X}_{t}\right), K_{f}\right)-F_{e}\right)
$$

Then, the following error dynamics is employed:

$$
\left(\ddot{X}_{t}-\ddot{X}_{t}^{d}\right)+\varsigma_{1}\left(\dot{X}_{t}^{d}-\dot{X}_{t}\right)+\varsigma_{2}\left(X_{t}^{d}-X_{t}\right)=0
$$

Now, the feedback linearizing control $P_{f l}$ is chosen to be

$$
P_{f l}=\frac{1}{A} M_{t}\left(\ddot{X}_{t}^{d}-\varsigma_{1}\left(\dot{X}_{t}^{d}-\dot{X}_{t}\right)-\varsigma_{2}\left(X_{t}^{d}-X_{t}\right)\right)-\frac{1}{A} C_{t}+\frac{1}{A} F_{e}+\frac{1}{A} R_{t}\left(x_{r}\right) \ddot{x}_{r}
$$

where $X_{t}^{d}, \dot{X}_{t}^{d}, \ddot{X}_{t}^{d}$ are the desired position, velocity, and acceleration and $\varsigma_{1}$ and $\varsigma_{2}$ are the control gains. In addition,Eq. (31) is uncertainty in the system, an auxiliary control input $w$ can be injected as follows

$$
\bar{P}_{f l}=P_{f l}+\frac{M_{t}}{A} w
$$

Using $\bar{P}_{f l}$ Eq. (32) yields the error dynamics

$$
\left(\ddot{X}_{t}-\ddot{X}_{t}^{d}\right)+\varsigma_{1}\left(\dot{X}_{t}-\dot{X}_{t}^{d}\right)+\varsigma_{2}\left(X_{t}-X_{t}^{d}\right)+\Theta(\cdot)-w=0
$$

where $\Theta(\cdot)$ is lumped uncertainty originating from the bounded uncertainties in the plant. Here, a layer of sliding manifold and a switching law on the reduced order manifold are defined so as to compensate for the bounded lumped uncertainty stemming from the difference between the actual and the nominal plant parameters [Acarman et al., 2001]. Therefore the layer of sliding manifold can be defined as

$$
S_{w}=\dot{e}_{t}+C_{w} e_{t}
$$

where $\dot{e}_{t}$ and $e_{t}$ denotes $\dot{X}_{t}-\dot{X}_{t}^{d}$ and $X_{t}-X_{t}^{d}$, respectively. It is noted that $C_{w}>0$. Now, let

$$
w=\left(\varsigma_{1}-C_{w}\right) \dot{e}_{t}+\varsigma_{2} e_{t}-\tilde{\mathrm{N}} \operatorname{sgn}\left(S_{w}\right)
$$

where $\tilde{N}>|\Theta(\cdot)|$. Then, $\dot{S}_{w}$ is expressed as

$$
\dot{S}_{w}=\ddot{e}_{t}+C_{w} \dot{e}_{t}=-\Theta(\cdot)-\tilde{\mathrm{N}} \operatorname{sgn}\left(S_{w}\right)
$$

Therefore, $\dot{S}_{w} \cdot S_{w}<0$ is achieved. In summary, the deburring system of interest is considered to have two subsystems as described. The interactive dynamics of the subsystems are decoupled in feedback sense by feedback linearization or Eq. (28) and suitable controllers are designed for the subsystems based on the motion coordination scheme as described. Then, a robust controller is designed for the tool subsystem to minimize the harmful effect of static and Coulomb frictions and nonlinear compliance of the 
pneumatic cylinder due to air compressibility. Therefore, the stability of the overall system can be achieved by properly selecting the feedback gains of each subsystem together with proper gains of the robust feedback for the tool as shown in Eqs. (33) - (36).

\section{Simulation}

Simulation study was performed to investigate the performance of the controllers developed for the robotic deburring systems with different tools: (1) the hybrid controller [12, 13, 20, 21, $22,23,24,28,39$ ] for the rigid tool based system (2) the coordination controller for the single active pneumatic cylinder tool (3) the coordination controller for the double active pneumatic tool based system. Note that the mathematical models for the rigid and the single active pneumatic cylinder tools are not shown due to their nature of being a subset of the IPAS.

Fig. 4 shows the simulation results for the hybrid control system. The following parameters were used in simulation:

$$
m_{1}=16 \mathrm{~kg}, m_{2}=12 \mathrm{~kg}, l_{1}=0.5 \mathrm{~m} \text {, and } l_{2}=0.7 \mathrm{~m}
$$

where $m_{1}$ and $m_{2}$ are the masses of each link of the 2 DOF manipulator, $l_{1}$ and $l_{2}$ are the lengths of each link. The feedback gains of the controller were chosen as following: $f_{d}=20 N, k_{p 1}=\operatorname{diag}[150,150,150], k_{d 1}=\operatorname{diag}[70,70,70], k_{p 2}=\operatorname{diag}[750,750,750]$, and $k_{d 2}=\operatorname{diag}[230,230,230]$ where $f_{d}$ is the desired force, and $k_{p i}$ and $k_{d i}(i=1,2)$ are the control PD gains.

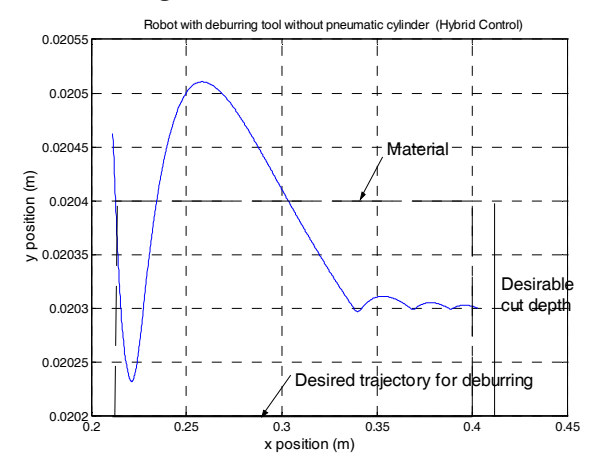

(a)

Fig. 4. Rigid tool (a) tracking (b) position error

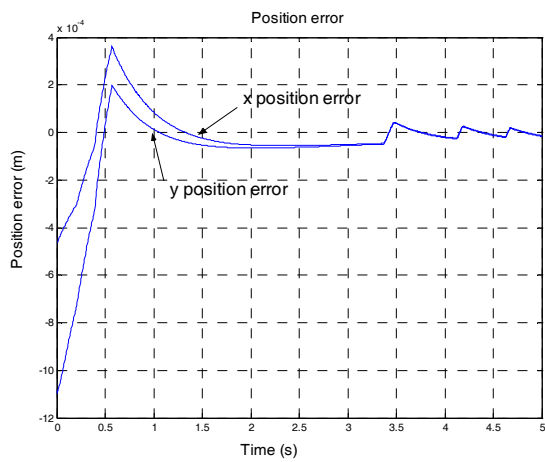

(b)

Fig. 4 (a) and (b) show the performance of the hybrid controller designed for the deburring robot with a rigid tool. In the simulation, the stiffness of the material was set to $500000 \mathrm{~N} / \mathrm{m}$ and the desired cut depth was chosen to be $0.0002 \mathrm{~m}$. The results show large deburring error, which remains oscillatory after large overshoot in the transient period due to chattering caused by the air compressibility and the contact motion between the robot and the workpiece. The following parameters were used for the tangential force as:

$$
b=16 \mathrm{~mm}, v_{t}=0.08 \mathrm{~m} / \mathrm{s} \text {, and } V_{t}=30,000 \mathrm{RPM} \text {. }
$$


Fig. 5 depicts the deburring performance of the coordination controller designed for the robot with a single active pneumatic cylinder tool. The following parameters were used for simulation:

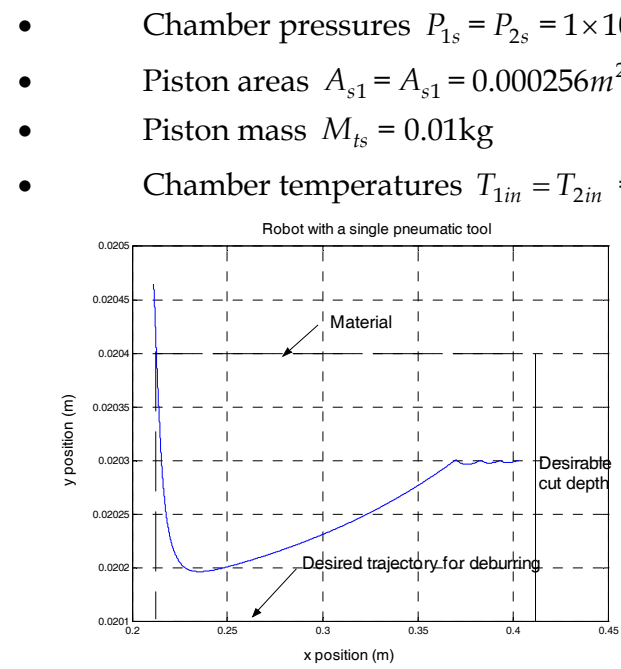

(a)

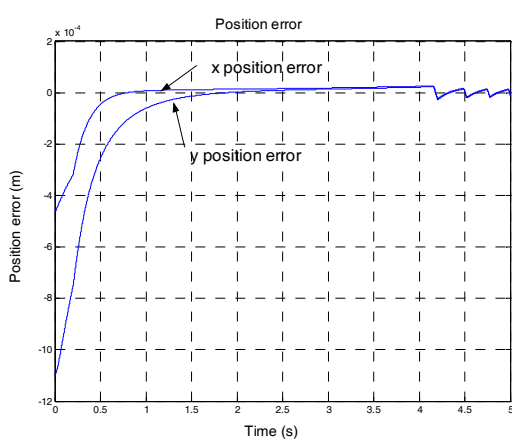

(b)

Fig. 5. Single pneumatic tool (a) tracking (b) position error

As shown in Fig. 5 (a) and (b), the transient performance is improved significantly with the single active pneumatic tool with the coordination controller in comparison to the previous case. However, the steady-state performance still remains unsatisfactory due to the chatter that appears in the response, which is caused by the compressibility of the air in the pneumatic cylinder and therefore requires repetitive deburring. Nevertheless, the simulation results demonstrate the potential of a pneumatic actuator as an efficient tool which can significantly enhance the performance of a deburring robot if the chattering effect can be eliminated or minimized by an improved design of the tool and/or an efficient control.

Fig. 6 demonstrates the deburring performance of the robot with the IPAS as shown in Fig. 1. The developed coordination control method by using feedback linearization was utilized for the IPAS based deburring system. It is noted that the initial position of $X_{t i}(i=1,2,3,4)$ is set to zero. The following is the additional parameters used for the integrated cylinder:

$$
\begin{gathered}
P_{3 j}=1 \times 10^{5} \mathrm{~Pa}, A_{1}=A_{2}=0.000256 \mathrm{~m}^{2}, A_{3}=A_{4}=0.00055 \mathrm{~m}^{2}, \mathrm{n}=0.8, F_{f 1,2}=10 \mathrm{~N}, F_{f 3,4}=15 \mathrm{~N}, \\
M_{t 1}=M_{t 2}=0.01 \mathrm{~kg}, M_{t 3}=M_{t 4}=0.015 \mathrm{~kg}, \text { and } T_{3 j}=293^{\circ} \mathrm{K} .
\end{gathered}
$$




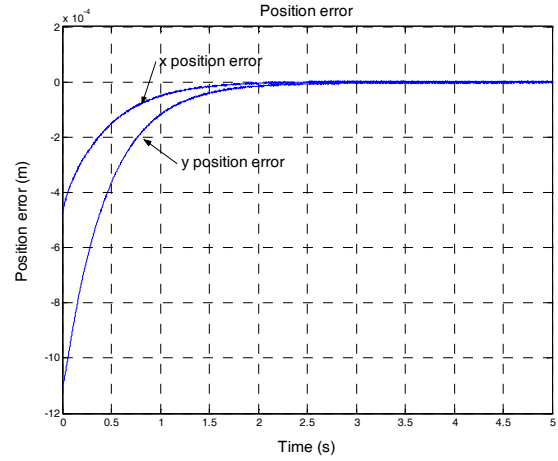

(a)

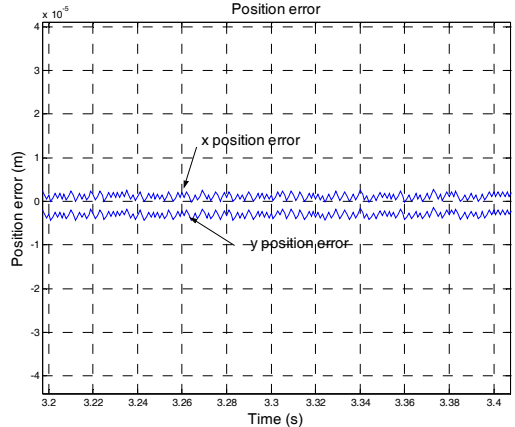

(b)

Fig. 6. Integrated double pneumatic cylinder (Coordinated control without robust control) (a) Position error (b) Enlarged position error

It is evident as shown in Fig. 6 (a) that the deburring performance of the system is greatly improved with the IPAS and the coordination controller. The simulation results show quick and smooth transient response and nearly zero steady-state error. The integrated system particularly improves the transient behavior in comparison to the single cylinder system. However, Fig. 6 (b) shows the chatter of position error of the IPAS when Fig. 6 (a) is enlarged. The chatter is from the compressibility and flexibility of the air. Such fluctuating position error can occur in harm of the system. Also, Combined with their limited damping and low stiffness properties, which arise from the compressibility of air, the accuracy and repeatability of the performance can be limited under variable payload and supply pressure. To eliminated and/or reduce the undesirable effect of nonlinearity, in next simulation, robust feedback linearization is employed.

Fig. 7 depicts the deburring performance of the coordination controller based on robust feedback linearization. The following parameters were used for simulation:

$$
\begin{gathered}
P_{3 j}=1 \times 10^{5} \mathrm{~Pa}, A_{1}=A_{2}=0.000256 \mathrm{~m}^{2}, A_{3}=A_{4}=0.00055 \mathrm{~m}^{2}, \mathrm{n}=0.8, F_{f 1,2}=10 \mathrm{~N}, F_{f 3,4}=15 \mathrm{~N}, \\
M_{t 1}=M_{t 2}=0.01 \mathrm{~kg}, M_{t 3}=M_{t 4}=0.015 \mathrm{~kg}, T_{3 j}=293^{\circ} \mathrm{K}, \varsigma_{1}=25, \varsigma_{2}=7, C_{w}=7, \tilde{N}=1, \text { and } \\
\Theta(\cdot)=0.5,
\end{gathered}
$$

Fig. 7 (b) shows the reduction of position error caused by the, which is caused by the compressibility of the air in the pneumatic cylinder. In this simulation, the oscillatory position errors are almost eliminated in difference with the previous results by using the robust feedback linearization. Through the robust feedback as shown in Fig. 3, the additional robust controller could soften the chatter by the air compressibility in pneumatic tool. The simulation results demonstrate the efficacy of the developed coordination control based on robust feedback linearization for the new deburring tool. 


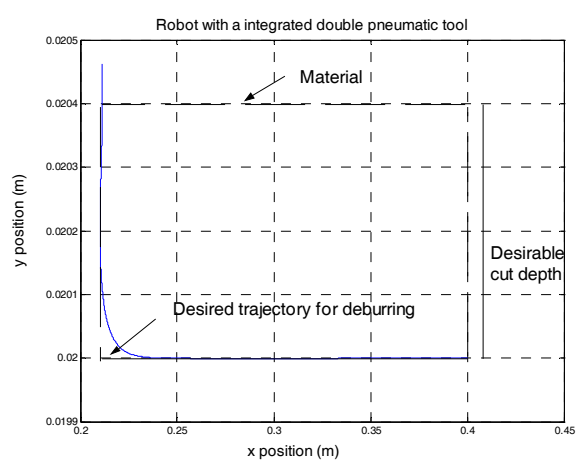

(a)

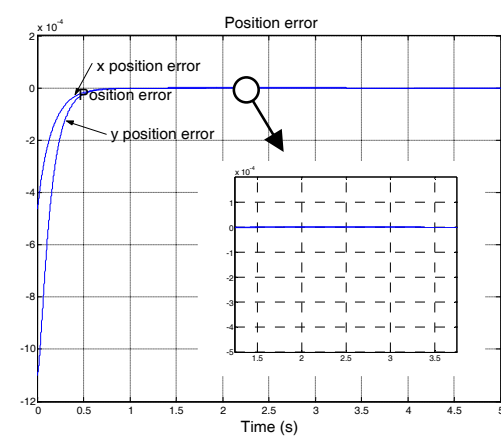

(b)

Fig. 7. Integrated double pneumatic cylinder (a) tracking (b) position error (Robust Feedback linearization)

\section{Conclusion}

High-quality robotic deburring requires efficient control of the deburring path and contact forces, as well as optimal selection of a suitable feed-rate and tool design. In this paper, an efficient robotic deburring method was developed based on a new active pneumatic tool, which considers the interaction among the tool, the manipulator, and the workpiece and couples the tool dynamics and a control design that explicitly considers deburring process information. A new active pneumatic tool was developed by physically integrating two pneumatic actuators, which implements double cutting action - initial cut followed by fine cut. Then, a control method was developed for the robotic deburring system based on the active pneumatic tool, which utilizes coordinated control based on a feedback linearization for the manipulator and a robust feedback linearization for the deburring tool using a pneumatic cylinder. From the simulation results, robust feedback linearization achieved the smooth transient response and nearly zero steady-state error in spite of the undesirable effect of external disturbances. The developed control system employs the two-level hierarchical control structure based on a simple coordination scheme. Simulation results showed that the developed system significantly reduces the chattering of the deburring robot and improves the deburring accuracy. Implementation of the developed method is intended for experimental verification in the future.

\section{References}

[1] Kuntze, H. B. (1984). On the closed-loop control of an elastic industrial robot, Proceedings: 1984 American Control Conference.

[2] Sharon A. \& Hardt D.E. (1984). Enhancement of robot accuracy using endpoint feedback and a macro-micro manipulator system, Proceedings: 1984 American Control Conference, pp.1836.

[3] Asada, H. \& Sawada, Y. (1984). Design of an adaptable tool guide for grinding robot, ASME Design Engineering Technical Conference paper, No. 84-Det-41. 
[4] Paul, F. W., Gettys, T.K. \& Thomas, J.D. (1982). Defining of iron castings using a robot positioned chipper, Proceedings: Robotics Research and Advanced Application, ASME, pp. 269-278.

[5] Sorli, M., Gastaldi, L., Codina, E., \& Heras, H. (1999). Dynamic analysis of pneumatic actuators, Elsevier Science.

[6]Armstmstrong-Helouvry, S., Dupont, P., \& Canudas De Wit, C. (1994). A survey of models, analysis tools and compensation methods for the control of machines with friction, Automatica, pp.1083-1183.

[7] Deccusse, J. \& Moog, C. H. (1985). Decoupling with dynamic compensation for strong invertible affine nonlinear systems, International Journal of Control, 42: 1387-1398.

[8] Isidori, A. (1985). Nonlinear control systems: An introduction, Springer verlag, Berlin, New york.

[9] Kazerooni, H. and Guo, J. (1987). Direct-drive, active compliant End-Effector, IEEE Journal of Robotics and Automation.

[10] Hollis, R.L. (1989). A planar XY robotic fine positioning device, Proceedings of the IEEE International Conference on Robotics and Automation, Raleigh, North Carolina.

[11]Raibert, M.H. \& Craig J.J. (1981). Hybrid position/ force control of manipulator," ASME Journal of Dynamics System, Measurements and Control, Vol.102, pp.126-133.

[12] Whitney, D. E. (1987). Historical perspective and state of the art in robot force control, International Journal of Robotics Research, vol. 6, no. 1, pp. 3-14.

[13] Bopp, T. (1983). Robotic finishing applications: Polishing sanding, grinding, Proceeding of the 13th International Symposium on Industrial Robots.

[14] Gustaffson, L. (1983). Deburring with industrial, Robots, Technical report, Society of Manufacturing Engineers.

[15] Hogan, N. (1984). Impedance control of industrial robots, Journal of Robotics and Computer Integrated Manufacturing, Vol. 1, No. 1, pp.97-113.

[16] Wang, D. \& Cheah, C. C. (1996). A robust learning control scheme for manipulators with target impedance at end-effectors, Robotics and Manufacturing: Recent Trends in Research and Applications, ASME Press, Vol. 6, pp. 851-856.

[17] R. Carelli, R. \& Kelly, R. (1991). An adaptive impedance/force controller for robot manipulators, IEEE Transactions on Automatic Control, Vol. 36, pp. 967-972.

[18] Yoshikawa, T., Sugie, T. \& Tanaka, M. (1988). Dynamic hybrid position/force control of robot manipulators: Controller design and experiment, IEEE Journal of Robotics and Automation, Vol. 4, pp. 699-705.

[19] Acarman, T., Hatipoglu, C. \& Ozguner, U. (2001). A robust nonlinear controller design for a pneumatic actuator, American Control Conference, 2001. Proceedings of the 2001, 25-27 June 2001, Vol.6, pp.4490 - 4495. 


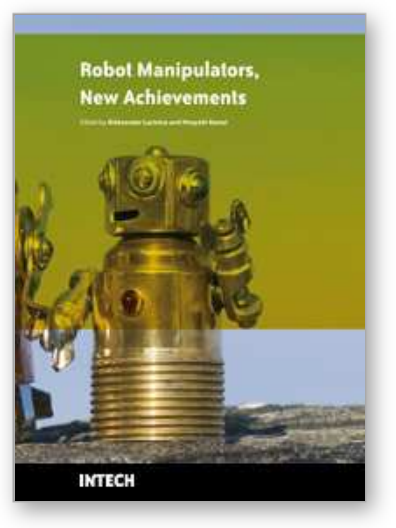

\author{
Robot Manipulators New Achievements \\ Edited by Aleksandar Lazinica and Hiroyuki Kawai
}

ISBN 978-953-307-090-2

Hard cover, 718 pages

Publisher InTech

Published online 01, April, 2010

Published in print edition April, 2010

Robot manipulators are developing more in the direction of industrial robots than of human workers. Recently, the applications of robot manipulators are spreading their focus, for example Da Vinci as a medical robot, ASIMO as a humanoid robot and so on. There are many research topics within the field of robot manipulators, e.g. motion planning, cooperation with a human, and fusion with external sensors like vision, haptic and force, etc. Moreover, these include both technical problems in the industry and theoretical problems in the academic fields. This book is a collection of papers presenting the latest research issues from around the world.

\title{
How to reference
}

In order to correctly reference this scholarly work, feel free to copy and paste the following:

Jae H. Chung (2010). Modeling and Control of a New Robotic Deburring System, Robot Manipulators New Achievements, Aleksandar Lazinica and Hiroyuki Kawai (Ed.), ISBN: 978-953-307-090-2, InTech, Available from: http://www.intechopen.com/books/robot-manipulators-new-achievements/modeling-and-control-of-anew-robotic-deburring-system

\section{INTECH}

open science | open minds

\section{InTech Europe}

University Campus STeP Ri

Slavka Krautzeka 83/A

51000 Rijeka, Croatia

Phone: +385 (51) 770447

Fax: +385 (51) 686166

www.intechopen.com

\section{InTech China}

Unit 405, Office Block, Hotel Equatorial Shanghai

No.65, Yan An Road (West), Shanghai, 200040, China

中国上海市延安西路 65 号上海国际贵都大饭店办公楼 405 单元

Phone: +86-21-62489820

Fax: +86-21-62489821 
(C) 2010 The Author(s). Licensee IntechOpen. This chapter is distributed under the terms of the Creative Commons Attribution-NonCommercialShareAlike-3.0 License, which permits use, distribution and reproduction for non-commercial purposes, provided the original is properly cited and derivative works building on this content are distributed under the same license. 